\title{
Tactile and Non-contact Sensors for Coordinate Measuring Technology
}

\author{
Dr.-Ing. habil. Ralf Christoph, Dr.-Ing. Ingomar Schmidt \\ Werth Messtechnik GmbH, Siemensstraße 19, 35394 Gießen, Germany \\ Ralf.Christoph@werth.de
}

\begin{abstract}
:
Coordinate measuring machines provide the most flexible technical solutions for dimensional measuring. Due to the possibility of combining several sensors in one machine, multisensor coordinate measuring machines are of particular advantage. When choosing the sensors for an application, the conditions of the measurement object, such as the dimensions of the features to be measured, surface characteristics as well as required accuracy, must be taken into consideration. The talk gives a short overview over the basic principles of available optical and tactile sensors for coordinate measuring technology. Realization examples for selected sensor principles in combination with modern multisensor coordinate measuring machines are presented and several application possibilities are explained. The focus is on optical sensors, which allow area measurements of workpiece surfaces and on special tactile sensors for microstructures.
\end{abstract}

Key words: Multisensor coordinate measuring machines, optical sensors, tactile optical sensors

\section{Introduction}

Coordinate measuring technology, especially micro and nano coordinate measuring technology, requires high precision measurements on increasingly smaller features distributed along parts of different sizes. Highest accuracies must be achieved for the dimensions of and the distances between different features. Another challenge is the measurement of parts with high aspect ratios, such as spray holes of injection nozzles.

To fulfill these requirements many different sensors are available. They can be classified according to the physical principles they use (Fig. 1). The variation of capabilities of these sensors requires the right sensor selection for each measuring task.

By using multisensor coordinate measuring machines, the sensor selection can be accomplished very comfortably and efficiently for a wide variety of measurement tasks. The advantages and the precision of the selected sensors can only be utilized in combination with a coordinate measuring machine with sufficient accuracy.

\section{Optical sensors}

High measuring speed, excellent lateral resolution and non-contact measuring with high accuracy are the well-known advantages of optical sensors [1].

Image processing sensors are suitable for lateral measurements, for example, the fast capturing of edges in transmitted and reflected light. Also, the scanning of contours to evaluate form tolerances and free form features is possible. Large geometries can be measured quickly and completely with high resolution using raster scanning. A large number of partial images are captured and combined into one high resolution image of the entire part. Using the "On the Fly" routine, the images and sensor positions are recorded during continuous machine travel resulting in the highest measurement speed. The ability to rapidly capture an enormous number of points makes image processing sensors the ideal choice for many applications.

If the surface topography must be determined, distance sensors are often used. In their basic form, these are single point sensors capable of measuring entire surfaces by scanning. Area sensors are capable of measuring many points on a surface in a single stationary position. 


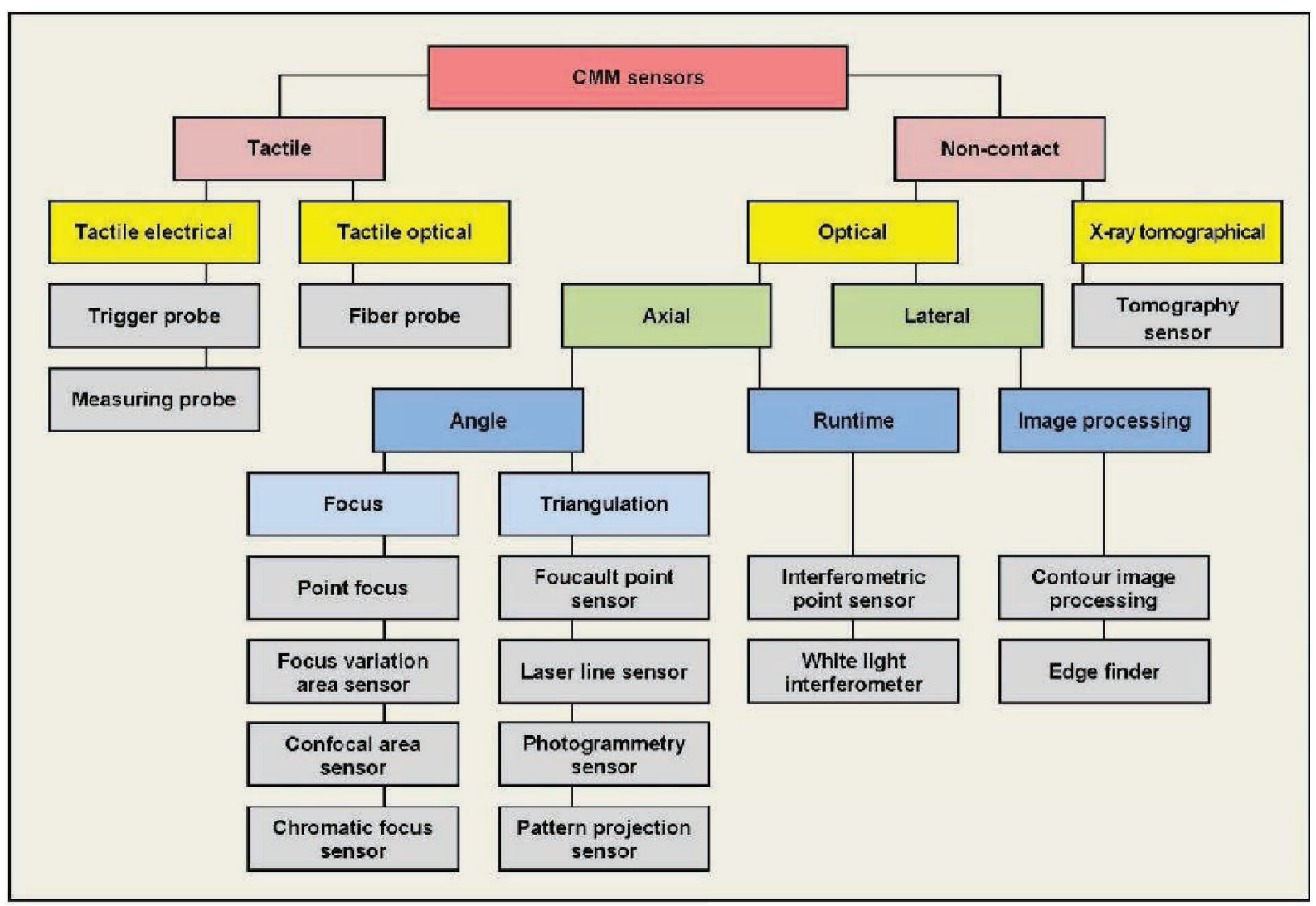

Fig. 1. Sensor classification according to physical principle [1].

The autofocus function of an image processing sensor, in its basic mode, can be used to measure single surface points by contrast evaluation. Its use is limited to structured and diffuse reflecting surfaces.

The Foucault single point distance sensor can scan partially reflective surfaces very fast, even with steep surface angles. Due to the long working distance, the risk of collisions is minimized. Typically, this type of triangulation sensor is integrated into the beam path of an image processing sensor.

Measurements on highly reflective surfaces (for example, optical surfaces or shiny molds) can be accomplished with chromatic focus sensors. These sensors use the wavelength dependence of the focal distance of optics.

Area sensors are available based on different optical principles. 3D-Patch is a more sophisticated autofocus function that uses the focus variation principle. Many points up to millions of focus points are measured in one cycle. The image is divided into a grid pattern or even down to single pixels. For each grid section or pixel the distance to the object surface is calculated separately. Like the basic autofocus, its application is limited to structured surfaces. Recent developments enable this kind of sensor to measure even on steeper and lower contrast surfaces. This is accomplished by the use of special lighting, high dynamic range (HDR) and image capturing with multiple illumination cycles (Fig. 2).
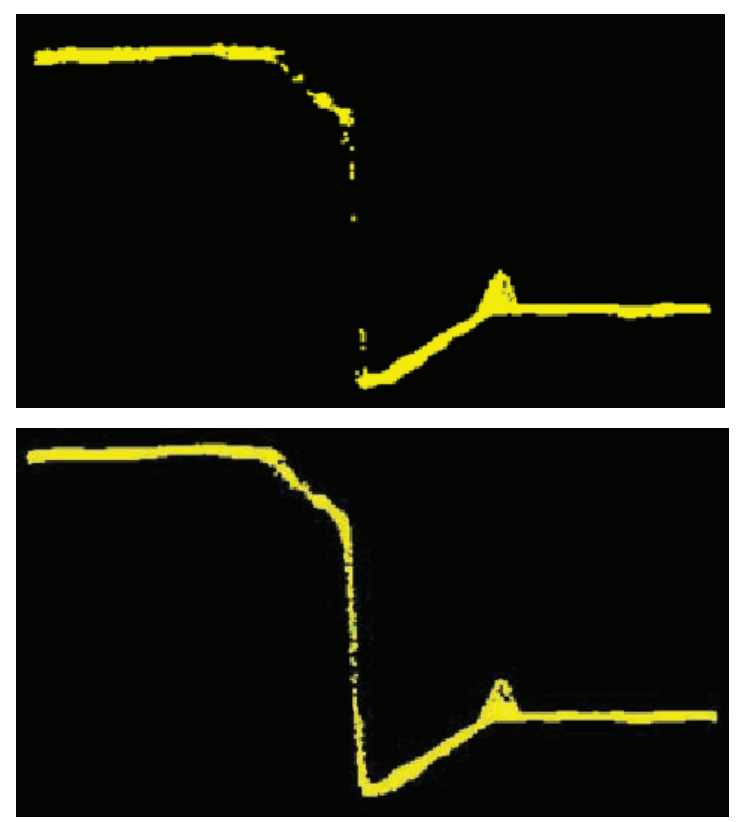

Fig. 2. Distribution of surface points measured on very steep edge with focus variation principle $3 D$ Patch (top-without HDR, bottom-with HDR function).

Another very accurate approach is the confocal microscope. These instruments use small pinholes on a rotating Nipkow Disk to truncate interfering light from neighboring object areas 
for each measurement point. This allows an intensity determination for each individual point. Surfaces with low or no contrast can be measured accurately. The maximum surface angle depends on the reflectivity and the aperture of the optics used. Application examples are shown in figure 3 .
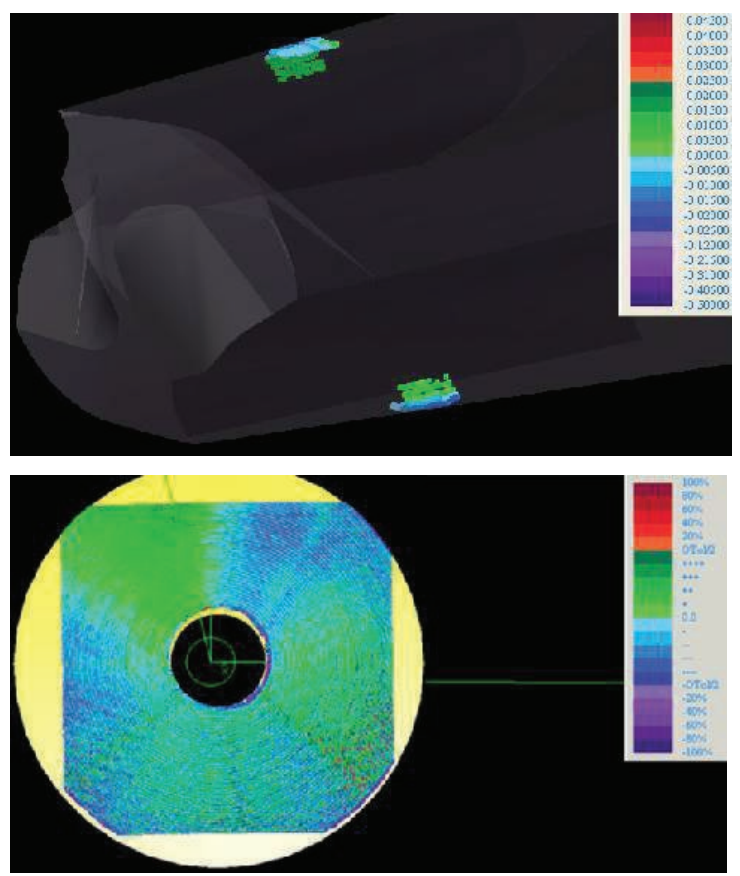

Fig. 3. Cutting edge radius (top) and topography and roughness of hydraulic valve seat (bottom), measured with the confocal "Nano Focus Probe".

\section{Tactile and tactile-optical sensors}

Compared to optical sensors, the advantage of tactile sensors is their extended 3D capability [2]. With tactile micro probes that use the conventional electro mechanical principles, the deflection of the probing sphere must be transmitted to a detection circuit via the stylus. This limits the smallest possible stylus diameter. In consequence, the miniaturization of the probing sphere diameter is practically limited to about $100 \mu \mathrm{m}$. The stiffness required for the mechanical signal transmission also limits the stylus length to a few millimeters. These stiff styli also cause high probing forces. Thus, easily deformed parts cannot be measured accurately. Because of the high Hertzian pressure (high probing force, small sphere size), sensitive features such as optical surfaces will be damaged by the probing process. Additionally, the risk of breaking the probe is generally very high. To monitor the probing process, an additional camera is required. Using these probes in a shop floor environment is not recommended and very difficult in laboratories as well. All these limitations create the need for alternative approaches to measure real micro structures such as small holes of injector nozzles or tools for micro molding.

So far, the most successful micro probe technology is the Werth Fiber Probe, with the advantage of the optical measurement of the sphere position [1]. The lateral deflection of the probing sphere is measured by an image processing sensor. The stylus does not need to transfer the probing forces and moments to a detection circuit. Thus, even for long styli, the diameter can be very small and probing sphere diameters down to $20 \mu \mathrm{m}$ are available. The small stylus diameter also provides very small probing forces in the micro-Newton range with the smallest measurement uncertainties. Flexible and sensitive parts can be measured with virtual non-contact. Because of its flexible stylus, the Fiber Probe is very resistant to breakage. In practice, the probe is frequently used in the shop floor environment for production process control. Depending on the working distance of the image processing sensor, stylus lengths up to $150 \mathrm{~mm}$ are possible. Micro features located up to $150 \mathrm{~mm}$ below the work piece surface can be measured without collision risk. Since the probing sphere is used for tactile contact and optical detection by the camera, the optical light path can be disturbed in small, deep holes by effects resulting from the penetration depth. This effect can be eliminated by adding a second sphere located higher on the fiber shaft. The second sphere does not penetrate the measured structure and is used for the optical imaging. Different fiber geometries can be combined in one coordinate measuring machine by using an automatic probe exchange rack.

This basic concept has been further developed to a full 3D sensor by using a fast displacement sensor for measuring the vertical deflection of the probe tip.

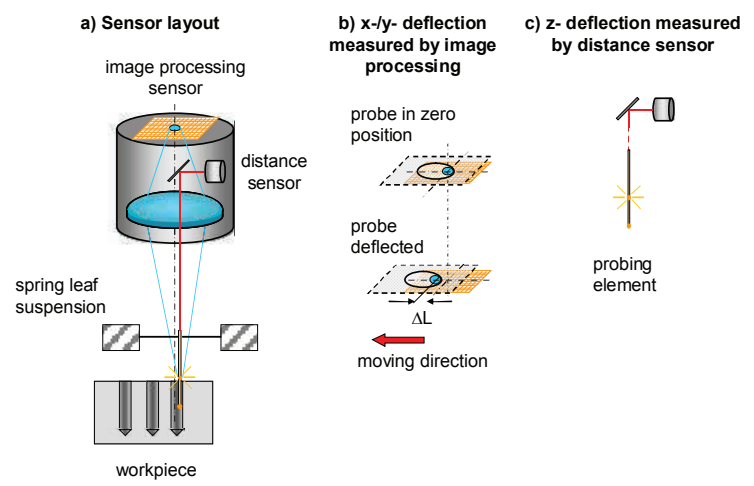

Fig. 4. 3D Fiber Probe for high precision three dimensional measurements of micro features: Measuring the lateral deflection of the probing sphere by image processing b) and the vertical deflection by a distance sensor c), combined within one optical system a). 
The distance sensor is integrated into the same optical beam path of the image processing sensor that is used to detect the lateral deflection. By using a thin spring leaf suspension for the stylus mounting, 3dimensional motions of the sphere with isotropic probing forces is provided (Fig. 4). As the stylus has to transmit only vertical deflections it can still be very small enabling probing sphere diameters down to $20 \mu \mathrm{m}$. Lateral and vertical deflection signals are combined by the software to provide the fast and accurate 3D scanning capabilities of conventional 3D scanning probes, but with micro spheres. An application example is shown in figure 5 . In another special application, an L-shaped stylus was manufactured to measure undercuts, for instance on a broach (Fig. 6), micro gears, small side bores, or even internal threads.

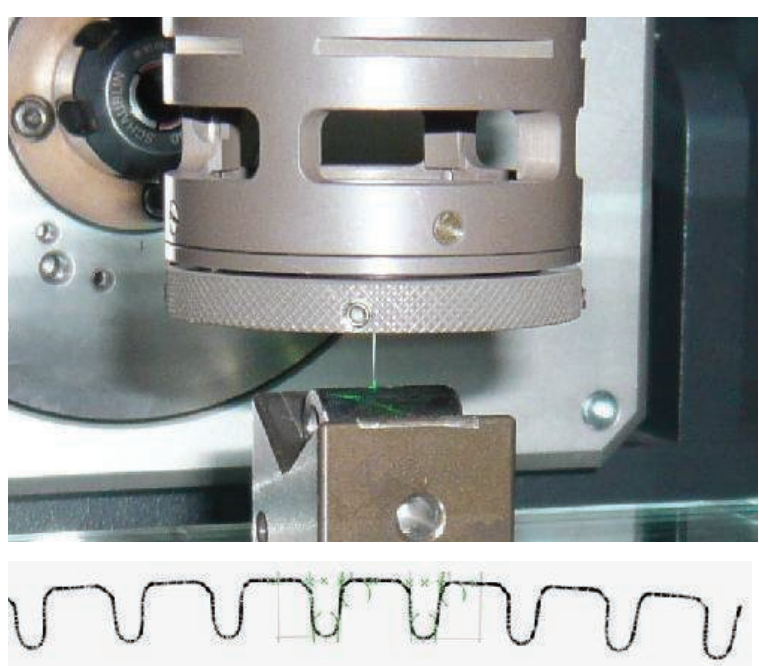

Fig. 5. Measuring the engravings on rollers (top) with the Werth 3D Fiber Probe: At a depth of $140 \mu \mathrm{m}$ a $30 \mu \mathrm{m}$ radius is measured at the root of a $80 \mu \mathrm{m}$ wide groove (bottom).

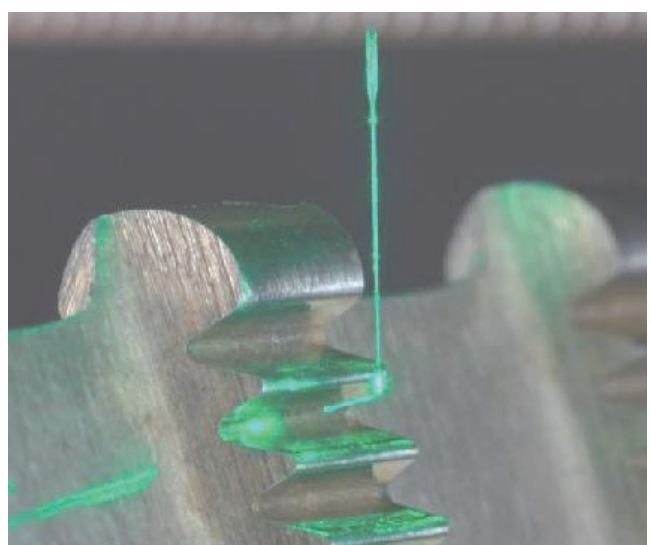

Fig. 6. Undercut measurement on a broach tooth form with the Werth 3D Fiber Probe in L-shape design.
Highest flexibility can be realized when different sensors are combined within a multisensor coordinate measuring machine. To enable the entire measurement range for combined measurements with different sensors a true "Multisensor" has been developed (Fig. 8). Its measuring head integrates the following sensors that are aligned in a common measurement axis and interchangeable with an automatic probe changer:

- Image processing sensor Werth Zoom

- Werth Laser Probe WLP

- Mechanical touch trigger and scanning probes

- Fiber Probe and 3D Fiber Probe WFP

- Werth Contour Probe WCP

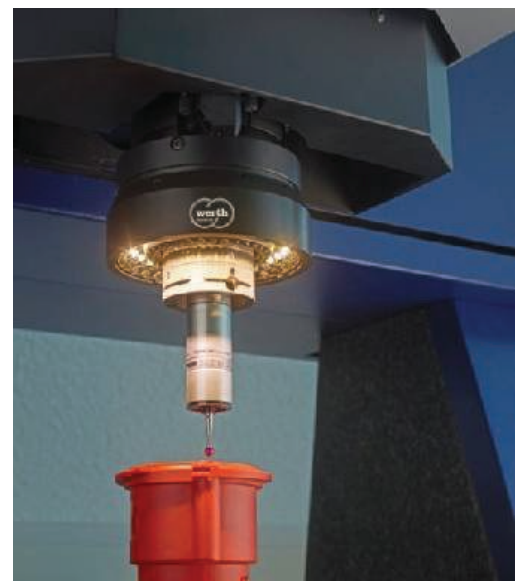

Fig. 7. "Multisensor"- The entire measurement

range is available for combined measurements. Different sensors can be precisely and automatically interchanged using parking stations.

\section{High precision multisensor coordinate measuring machines}

As mentioned above, the advantages and the precision of the selected sensors can only be utilized in combination with a coordinate measuring machine with sufficient accuracy.

Coordinate measuring machines used to measure micro geometries must be able to measure with very high repeatability and very small absolute errors. Primarily, the accuracy is influenced by the machine axes and the quality of geometric correction. Furthermore, the environmental conditions, especially the temperature and mechanical vibrations, have significant influence.

All these requirements are addressed in the ultra accurate coordinate measuring machine VideoCheck UA based on Werth's proven CMM technology. Reproducibility is enabled by a scale resolution of $0.5 \mathrm{~nm}$ and optimized 
mounting of the scales to separate them from all temperature influences. This completely utilizes the advantage of zero thermal expansion of the Zerodur scales. A stable granite base and active isolation systems for vertical and horizontal directions reduce vibrations. A sophisticated coupling between the drive systems and machine axes is applied to reduce systematic measurement errors. This proven design results in a measurement reproducibility down to a range of $10 \mathrm{~nm}$ (equivalent to a standard deviation of about $2 \mathrm{~nm})$. The specification of the maximum permissible error (MPE) for E1 unidirectional is $(0.15+L / 900) \mu \mathrm{m}$, the value of MPE for $E$ is $(0.25+\mathrm{L} / 600) \mu \mathrm{m}$. Due to the stable machine base, the vertical measurement range is available with $250 \mathrm{~mm}$ (Fig. 8). Thus, fixtures, rotary axes and large parts with micro features can be measured within the measurement volume. To achieve these specifications, a probing system with appropriately small probing errors is required.

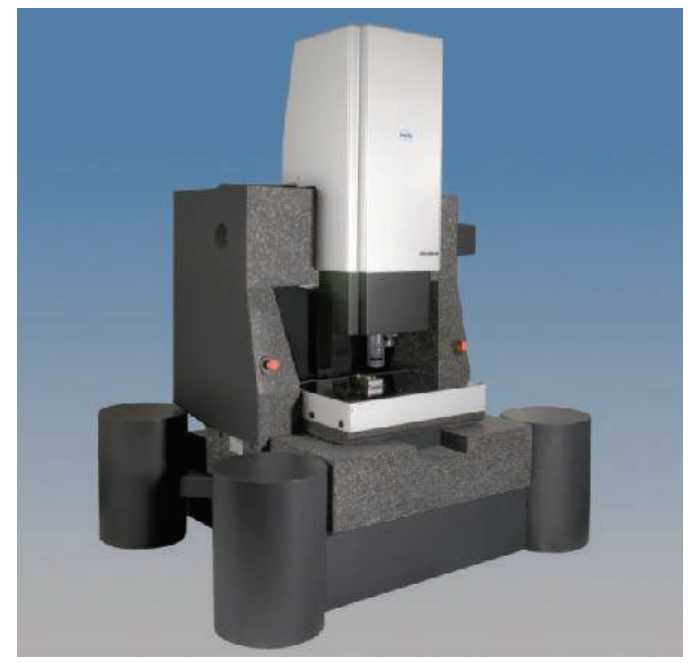

Fig. 8. Ultra high accuracy multisensor coordinate measuring machine Werth VideoCheck UA with $3 D$ Fiber Probe, high precision image processing sensor, laser probe and chromatic focus probe (additional sensor combinations are possible).

\section{References}

[1] Christoph, Ralf; Neumann, Hans Joachim: Multisensor-Koordinatenmesstechnik. München: Süddeutscher Verlag onpact, 2013. (Die Bibliothek der Technik, Band 352)

[2] A. Weckenmann (Hrsg.):

Koordinatenmesstechnik - Flexible Strategien für funktions- und fertigungsgerechtes Prüfen, 2., vollständig überarbeitete Auflage - Carl Hanser Verlag München Wien, 2012, ISBN 978-3-44640739-8 